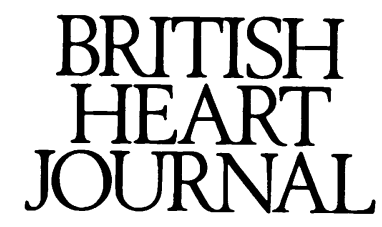

\title{
Concerning falsely negative and falsely positive electrocardiographic responses to exercise
}

The recognition that the progression of coronary artery disease can be slowed or even reversed by risk factor modification, that in some discrete subsets of patients revascularisation can prolong life as well as relieving symptoms, and that myocardial ischaemia may be symptomatically silent has led to an increased commitment to diagnosing ischaemic heart disease before the onset of ischaemic events. To accomplish this goal, exercise tests employing one of several protocols are being used with increasing frequency by family physicians, internists, and cardiologists not only to determine the causes of chest discomfort and the presence of residual ischaemia after a myocardial infarction but also to screen patients for the likelihood of ischaemia. Often the results of such exercise tests are used to determine whether or not other studies and procedures, particularly cardiac catheterisation and revascularisation, are indicated. For this reason an understanding of factors that can cause either falsely negative or falsely positive results has assumed increasing importance.

\section{Effects of probability}

It is now generally recognised that the probability of coronary artery disease in the population being screened affects the interpretation of the exercise electrocardiogram (ECG). ${ }^{1}$ In a population with a low probability of ischaemic heart disease, a negative test is more likely to be a true negative whereas a test meeting the criteria for positivity would be suspect. Conversely, in those with a high probability of ischaemic heart disease a positive test is more likely to be an indication of ischaemia (and coronary artery disease) and a negative test would be suspect. For this reason, factors capable of giving both false negative and false positive ECG responses must be appreciated and considered, particularly in patients in whom the result is contrary to that predicted by the likelihood of the disease. Indeed, even in patients in whom the result is as predicted by the probability of disease, it is important to appreciate that the specificity and sensitivity of the exercise test when referenced to coronary anatomy is between $75 \%$ and $85 \% .{ }^{23}$ Thus even in these patients myocardial ischaemia may occur in the absence of typical ECG changes and, in some situations, ECG changes characteristic of ischaemia may occur in the absence of myocardial ischaemia.

\section{Falsely negative responses}

Myocardial ischaemia may occur in the absence of changes in the body surface ECG during coronary angioplasty. In these patients the electrogram recorded over the ischaemic area during balloon inflation by extending the guide wire beyond the area of obstruction and connecting it to the $\mathrm{V}$ lead terminal will show marked ST segment changes while the simultaneously recorded body surface ECG often shows no change. ${ }^{4}$ There are several explanations for the phenomenon of absent ECG changes in the setting of myocardial ischaemia. (a) The location of ischaemia may be electrically silent relative to the body surface ECG. This is particularly true if the ischaemia is within the distribution of the circumflex coronary artery. ${ }^{5}$ (b) The magnitude of the ischaemic zone may be such that the solid angle created across the ischaemic margins from the body surface leads is inadequate to generate ST segment changes on the body surface. ${ }^{67}$ This is probably the mechanism underlying the dichotomy between the changes in the local electrogram and the body surface electrocardiogram during angioplasty. (c) An area of ischaemia may be so diffuse that the electrical gradients oriented in one direction will be cancelled by electrical gradients oriented in the opposite direction. ${ }^{8}$ This may occur in the setting of left main coronary artery disease. However, in this setting, one would expect that other findings such as a decrease in exercise tolerance, a fall in blood pressure during exercise, or the development of auscultatory findings suggestive of ventricular dysfunction - that is, a third sound or a murmur of mitral insufficiency after exercise-would suggest myocardial ischaemia. For this reason it is clearly important to incorporate these variables into the evaluation of the patient's response to exercise. The actual incidence of false negative ECG changes in patients either with or without a high pre-test likelihood of ischaemia is not known because in most studies the result is compared with the anatomical findings determined by coronary angiography rather than with the presence of ischaemia. In one recent study which compared the exercise ECG with coronary artery flow reserve ${ }^{9}$ three of 17 patients with abnormal flow reserve had normal ECG exercise tests while three of 23 patients with normal flow reserve had abnormal exercise tests. In another study that compared cross sectional exercise echocardiograms with exercise electrocardiograms ${ }^{10}$ nine of 13 patients with $>50 \%$ obstruction of one or more coronary arteries showed normal exercise ECGs but abnormal exercise echocardiograms.

\section{Falsely positive responses}

Various factors can also cause ECG changes with exercise that meet the standard criteria for an ischaemia response-that is, horizontal or downsloping ST segment depression $\geqslant 100 \mu \mathrm{V}$ measured $80 \mathrm{~ms}(0.80 \mathrm{~s})$ after the junction of the QRS complex and the onset of the ST segment (the $\mathrm{J}$ point)-even in the absence of exercise 


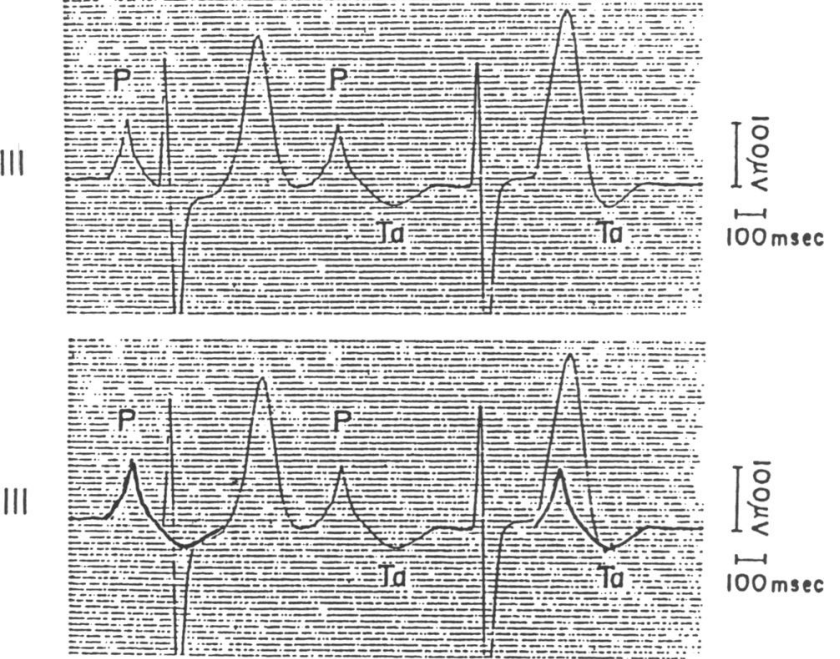

Example of false ST segment depression caused by atrial repolarisation in a patient with high grade atrioventricular block. Reprinted with permission from the American Heart Fournal. ${ }^{14}$

induced myocardial ischaemia or ECG changes at rest. These include agents that alter the plateau phase or the phase of rapid repolarisation of the action potential or both and by this mechanism lead to the development of electrical gradients which cause ST segment changes on the body surface ECG. Examples include, but are not limited to, hypokalaemia, drugs that directly affect the myocardial cell - that is, digitalis and the type I and III antiarrhythmic drugs - and left ventricular hypertrophy. ${ }^{11}$

\section{Effects of atrial repolarisation}

An additional factor that may affect the ST segment is the atrial repolarisation wave or the atrial ST segment and $T$ wave. Most medical students and house officers realise that there should be an atrial ST segment and T wave but will state incorrectly that the atrial $T$ wave is located within QRS complex. Most often, there is little appreciation of the magnitude, direction, or duration of the atrial repolarisation wave or that the atrial ST and T wave may cause electrocardiographic changes in the ST segment that are indistinguishable from those caused by ischaemia. The potential impact of atrial repolarisation on the ST segment is appreciated by those with an interest in electrocardiography ${ }^{1213}$ and the characteristics of atrial repolarisation have been studied in laboratory animals and in humans. ${ }^{12} 13$ It has been shown that the atrial ST segment and $\mathrm{T}$ wave are usually opposite in direction to the $\mathbf{P}$ wave and of significant magnitude, with the area under the atrial $\mathrm{T}$ wave roughly equivalent to the area under the $\mathrm{P}$ wave. In humans the duration of the interval from the onset of the $P$ wave to the end of the atrial $T$ wave averages $450 \mathrm{~ms}$ at a rate of $70 \mathrm{bpm}^{14}$ but may last as long as $600 \mathrm{~ms} .{ }^{15}$ Normally, the interval from the onset of the $\mathrm{P}$ wave to the end of the QRS complex is approximately $240 \mathrm{~ms}$ ( $P R=160 \mathrm{~ms}, Q R S=80 \mathrm{~ms}$ ). Thus approximately $200 \mathrm{~ms}$ of the atrial ST and T wave will extend beyond the end of the QRS complex. Because the interval from the end of the QRS complex to the end of the $T$ wave is in the range of $300 \mathrm{~ms}$, it is apparent that the atrial ST and T wave will extend well into the middle portion of the ventricular $T$ wave. In those leads with a positive $P$ wave, the downsloping nature of the atrial ST segments and the inverted atrial $\mathrm{T}$ wave may cause ST segment depression which is indistinguishable from that caused by ischaemia. The accompanying figure from an article by Hayashi et al shows an example of this effect. The upper panel shows lead III from a patient with high grade atrioventricular block and illustrates a positive $\mathrm{P}$ wave with a negative atrial $\mathrm{T}$ wave (Ta). A P wave occurs just before the first QRS complex and the ST segment is depressed. The second P-Ta complex is completed before the second QRS complex and the ST segment is not depressed. In the lower panel the entire P-Ta complex is superimposed on the first QRS complex, and illustrates that the ST segment depression is due to the atrial repolarisation wave. The onset of the third P-Ta complex coincides with the onset of the second $T$ wave and is responsible for the rather acute angle between the ST segment and the $T$ wave.

The role of atrial repolarisation in causing false positive electrocardiographic responses to exercise was recently the topic of an article, editorial, and exchange of letters in the fournal of the American College of Cardiology. ${ }^{12} 1316$ In this study, patients with exerciseinduced ST segment depression meeting criteria for ischaemia, but in whom ischaemia was viewed as unlikely on the basis of cardiac catheterisation or radionuclear studies, were compared with a matched group of patients in whom similar exercise-induced ST segment changes were attributed to ischaemia. The patients with a false positive exercise test were characterised by markedly downsloping PR segments at peak exercise which reflected the atrial ST segment. They were also identified by a longer exercise time and more rapid peak heart rates than patients in whom the ST segment depression was the result of ischaemia. This reflected the increase in the amplitude of the $\mathrm{P}$ wave and, in the opposite direction, of the amplitude of the atrial $T$ wave induced by the increase in heart rate. The third factor identifying the patients with a false positive exercise test was the absence of exercise-induced chest pain. Patients with a false positive exercise test were also noted to have taller $P$ waves during exercise and a greater augmentation of the $P$ wave amplitude during exercise than patients with a truly positive test. In this retrospective study the combination of a downsloping PR segment in two of the three inferior leads (II, III, and/or aVF) and either an exercise duration of more than 4 minutes or heart rates at peak exercise of more than $125 \mathrm{bpm}$ identified the false positive responders with a sensitivity of $84 \%$ and a specificity of $87 \%$.

There is no reason to expect that patients with exercise-induced myocardial ischaemia will be any less liable to the influence of atrial repolarisation or to the other factors capable of causing a false positive test. The point is that ECG changes or their absence associated with exercise should not, by themselves, be considered an absolute marker for the presence or absence of ischaemia. As with any other test the results must be interpreted within the context of the clinical presentation and the historical and physical findings. As screening for coronary artery disease becomes more prevalent and as those with less and less experience in performing and interpreting the electrocardiographic response to exercise participate in this method of screening it will become increasingly important to recognise the potential mischief an incorrect interpretation can cause and the factors that may lead to both falsely negative and falsely positive results.

LEONARD S GETTES

Division of Cardiology,

University of North Carolina at Chapel Hill,

Chapel Hill, NC 27599, USA

PETER SAPIN

University of Kentucky,

Lexington, $K Y$ 40536, USA

1 Diamond GA, Forrester JS. Analysis of probability as an aid in the clinical

diagnosis of coronary artery disease. N Engl f Med 1979;300:1350-8.

Gianrossi R, Detrano R, Mulvihill D, et al. Ex disease: A meta-analysis. sion in the diagnosis of

3 Melin JA, Piret LJ, Vanbutsele RJM, et al. Diagnostic value of exercise lin JA, Piret L J electrocardiography and thallium myocardial scintigraphy in pateach without previous myocardial 
4 Jain A, Gettes LS. Patterns of ST-segment depression change during acute no-flow myocardial ischemia produced by balloon occlusion during angioplasty of the left anterior descending coronary artery. $A m$ Cardiol 1991;67:305-7.

5 Huey BL, Beller GA, Kaiser DL, Gibson RS. A comprehensive analysis of myocardial infarction due to left circumflex artery occlusion: comparison with infarction due to right coronary artery and left anterior descending artery occlusion. F Am Coll Cardiol 1988;12:1156-66.

6 Holland RP, Brooks H. TQ-ST segment mapping: Critical review and analysis of current concepts. Am $\mathcal{F}$ Cardiol 1977;40:110-29.

7 Cook RW, Edwards JE, Pruitt RD. Electrocardiographic changes in acute subendocardial infarction: II Small subendocardial infarctions. Circulation 1958;178:613-22.

8 Autenrieth G, Surawicz B, Kuo CS, Arita M. Primary T wave abnormalities caused by uniform and regional shortening of ventricular monophaties caused by uniform and regional shortening of vent
sic action potential in dog. Circulation 1975;51:668-76.

9 Wilson RF, Marcus ML, Christensen BV, Talman C, White CW. Accuracy of exercise electrocardiography in detecting physiologically significant coronary arterial lesions. Circulation 1991;83:412-21.
10 Armstrong WF, O'Donnell J, Dillon JC, McHenry PL, Morris SN, Feigenbaum $\mathrm{H}$. Complementary value of two-dimensional exercise
echocardiography to routine treadmill exercise testing. Ann Intern Med 1986;105:829-35.

11 Detrano R, Froelicher VF. Exercise testing: Uses and limitations considering recent studies. Prog Cardiovasc Dis 1988;31:173-204.

12 Sapin PM, Koch G, Blauwet MB, McCarthy J, Hinds SW, Gettes LS. Identification of false positive exercise tests with use of electrocardiographic criteria: A possible role for atrial repolarization waves. $\mathcal{F} \mathrm{Am} \mathrm{Coll}$ Cardiol 1991;18:127-35.

13 Ellestad MH. Role of atrial repolarization in false positive exercise tests. $\mathcal{F}$ Am Coll Cardiol 1991;18:136-7.

14 Hayashi $\mathrm{H}$, Okajima M, Yamada $\mathrm{K}$. Atrial $\mathrm{T}(\mathrm{Ta})$ wave and atrial gradient in patients with A-V block. Am Heart $\mathcal{F}$ 1976;91:689-98.

15 Sivertssen $\mathrm{E}$. The atrial recovery wave $(\mathrm{Pt})$ studied by averaging computer technique in patients with complete heart block. $f$ Electrocardiol 1972;5: 243-52.

16 Soloff LA. Electrocardiographic criteria for identifying false positive tests. f Am Coll Cardiol 1992;19:1677-8. 